\title{
Concise Caching of Driving Instructions
}

\author{
Jeppe Rishede Thomsen Man Lung Yiu \\ Hong Kong Polytechnic University \\ \{csjrthomsen,csmlyiu\}@comp.polyu.edu.hk
}

\author{
Christian S. Jensen \\ Aalborg University \\ csj@cs.aau.dk
}

\begin{abstract}
Online driving direction services offer fundamental functionality to mobile users, and such services see substantial and increasing loads as mobile access continues to proliferate. Cache servers can be deployed in order to reduce the resulting network traffic. We define so-called concise shortest paths that are equivalent to driving instructions. A concise shortest path occupies much less space than a shortest path; yet it provides sufficient navigation information to mobile users. Then we propose techniques that enable the caching of concise shortest paths in order to improve the cache hit ratio.

Interestingly, the use of concise shortest paths in caching has two opposite effects on the cache hit ratio. The cache can accommodate a larger number of concise paths, but each individual concise path contains fewer nodes and so may answer fewer shortest path queries. The challenge is to strike a balance between these two effects in order to maximize the overall cache hit ratio. In this paper, we revisit two classes of caching methods and develop effective caching techniques for concise paths. Empirical results on real trajectory-induced workloads confirm the effectiveness of the proposed techniques.
\end{abstract}

\section{Categories and Subject Descriptors}

H.2.8 [Database Management]: Database Applications-Spatial databases and GIS

\section{General Terms}

Algorithms, Performance

\section{Keywords}

road networks, shortest paths, caching

\section{INTRODUCTION}

Navigation services offer step-by-step navigation, which provide a driver with driving instructions ${ }^{1}$ based on the driver's current GPS location [7]. We illustrate such driving instructions in

\footnotetext{
${ }^{1}$ These instructions can be spoken or given visually.

Permission to make digital or hard copies of all or part of this work for personal or classroom use is granted without fee provided that copies are not made or distributed for profit or commercial advantage and that copies bear this notice and the full citation on the first page. Copyrights for components of this work owned by others than ACM must be honored. Abstracting with credit is permitted. To copy otherwise, or republish, to post on servers or to redistribute to lists, requires prior specific permission and/or a fee. Request permissions from Permissions@acm.org.

SIGSPATIAL'14, November 04 - 07 2014, Dallas/Fort Worth, TX, USA

Copyright 2014 ACM 978-1-4503-3131-9/14/11 ...\$15.00

http://dx.doi.org/10.1145/2666310.2666401.
}

Figure 1. Suppose that the driver starts at node $v_{1}$ and the target is node $v_{10}$. First, a detailed model uses point-by-point instructions, which essentially form the shortest path: $S P_{1,10}=$ $\left\langle v_{1}, v_{3}, v_{4}, v_{5}, v_{7}, v_{9}, v_{10}\right\rangle$. In contrast, a concise model uses turn-by-turn instructions that are necessary for navigation, e.g., $\left\langle v_{1}, \Uparrow v_{5}, \Gamma^{90} \Uparrow v_{10}\right\rangle$. These instructions combine a sequence of checkpoints (e.g., $\left.v_{1}, v_{5}, v_{10}\right)$ and turn instructions (e.g., $\Uparrow, \Gamma^{90}$ ). After leaving $v_{1}$, the user should continue (indicated by $\Uparrow$ ) until reaching a checkpoint $\left(v_{5}\right)$. Finally, the user should make a right turn $\left(\underline{\Gamma^{90}}\right)$ and then continue ( $(\underline{)})$ until reaching the target $\left(v_{10}\right)$.

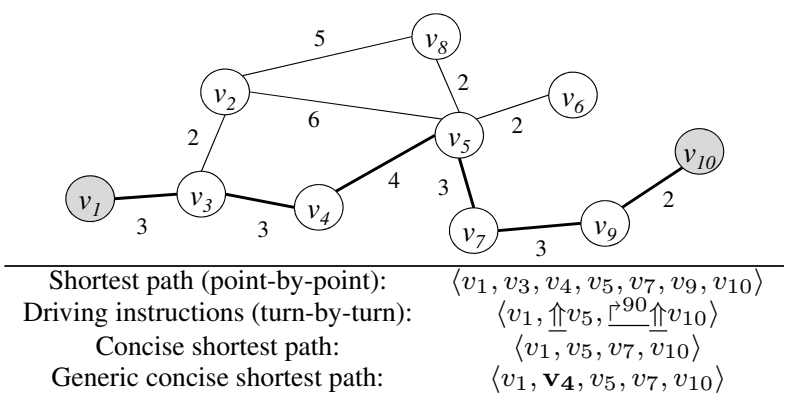

Figure 1: A road network, with a query from $v_{1}$ to $v_{10}$

Mobile users are increasingly using online driving direction services (rather than offline navigation software) as they are free of charge and do not require purchase and installation of up-to-date map data on mobile devices. Examples of driving direction APIs include Google Directions [1] and MapQuest Directions [2]. They can provide users with both shortest paths and turn-by-turn driving instructions.

The above services receive intensive workloads from mobile users on a daily basis. They can deploy web cache servers in order to reduce the network traffic $[3,19]$. In our application context, we propose to cache driving instructions at web cache servers. For the sake of cache management, we represent concise driving instructions (with checkpoints and turn instructions) according to a unified format called concise shortest path. By replacing turn instructions by nodes, we obtain the following concise shortest path for our example: $C P_{1,10}=\left\langle v_{1}, v_{5}, v_{7}, v_{10}\right\rangle$. The default driving instruction is to go straight until reaching the next node in the concise shortest path (e.g., from $v_{1}$ to $\left.v_{5}\right)$. Upon reaching such a node $\left(v_{5}\right)$, the driver checks whether the next such node $\left(v_{7}\right)$ is adjacent; if yes, the user makes a turn there. We elaborate on the construction of and navigation using such paths in Section 4. Figure 2 shows examples of a shortest path $S P$ (crosses) and the corresponding concise shortest path $C P$ (dots).

The use of concise shortest paths in caching has two opposite ef- 


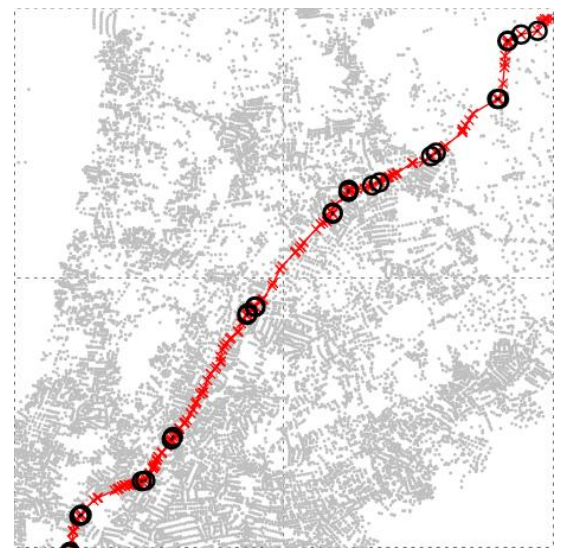

Figure 2: Shortest path (crosses) and concise shortest path (circles), in New York

fects on the cache hit ratio. Clearly, the cache can accommodate a larger number of concise paths than corresponding complete paths, suggesting a higher cache hit ratio. However, a concise path contains fewer nodes than its corresponding complete path and can answer fewer shortest path queries than the complete path. Consider the concise shortest path $\left\langle v_{1}, v_{5}, v_{7}, v_{10}\right\rangle$ in Figure 1. By the optimal subpath property [8], this path can answer any query whose end node lies on the path. Thus, this path can answer $Q_{5,10}$ (the query from $v_{5}$ to $v_{10}$ ) but not $Q_{4,10}$ (the query from $v_{4}$ to $v_{10}$ ).

Intuitively, if a query (say, $Q_{4,10}$ ) is frequent, then it is desirable to include its query nodes into a concise path. We use the term generic concise shortest path for a shortest path that extends a concise shortest path to include such frequent nodes. An example is the path $\left\langle v_{1}, \mathbf{v}_{\mathbf{4}}, v_{5}, v_{7}, v_{10}\right\rangle$ as shown in Figure 1. Although such a path is slightly less compact than $C P$, it can answer one more frequent query (e.g., $Q_{4,10}$ ) than $C P$. In general, there are $2^{|S P|-|C P|}$ possible instances of generic shortest paths having the same start and end nodes. Which of them should be cached? Our key challenge is to select generic shortest paths such that the overall cache hit ratio is maximized.

Our contributions are:

- We propose the notion of a generic concise shortest path that enables a trade-off between the path size and the number of queries answerable by the path.

- We present a statistics-driven model for selecting generic concise paths in a static caching setting.

- We develop an adaptive technique for determining generic concise paths in a dynamic caching setting.

The outline of the paper is as follows. Section 2 reviews related work, and Section 3 describes the problem setting. Next, we introduce concise shortest paths in Section 4, and we present caching techniques for them in Sections 5 and 6. Then, we evaluate the performance of our proposed techniques in Section 7. Finally, Section 8 offers conclusions.

\section{RELATED WORK}

\section{Compact representations of shortest paths.}

We proceed to review existing work on compressing a shortest path, i.e., representing it with few nodes.
The K-skip shortest path [21] is a path that contains at least one out of every $\mathrm{K}$ consecutive nodes in the corresponding shortest path. In Figure 1, the 3-skip shortest path of $S P_{1,10}$ is: $S K I P_{1,10}=\left\langle v_{1}, v_{5}, v_{10}\right\rangle$. Unfortunately, unlike concise shortest paths, K-skip shortest paths are lossy and may provide ambiguous driving instructions to the user. In practice, during driving, only a small portion of the road network is within the driver's eyesight. For example, when the user reaches $v_{3}$, the above 3-skip path $S K I P_{1,10}$ does not contain sufficient information to decide whether to go to $v_{2}$ or to $v_{4}$.

Batz et al. [6] develop a more effective compression method for shortest paths by using a shortest path index. However, this method requires the introduction of a shortest path index at the client side in order to decode the compressed path correctly. Otherwise, the client cannot obtain unambiguous driving instructions.

\section{Semantic caching.}

Caching has been studied extensively for database systems [17] and web search engines [16]. In the traditional caching model, each query requests a specific data item, and the cache only supports exact matches. On the other hand, in the semantic caching model $[12,14,22,24]$, each cached query result (or result set) is associated with a validity range, and it can be used to answer and refine any query that intersects its validity range. Semantic caching has been studied for spatial queries $[12,14,24]$ and for shortest path queries [22].

In previous work [22], we have studied the semantic caching of shortest paths. By the optimal subpath property [8], a shortest path $S P$ can answer any shortest path query $Q_{s, t}$ whose source $s$ and target $t$ both fall into $S P$. For example, in Figure 1, the shortest path $S P_{1,10}=\left\langle v_{1}, v_{3}, v_{4}, v_{5}, v_{7}, v_{9}, v_{10}\right\rangle$ can answer the shortest path query $Q_{4,10}$ as path $S P_{1,10}$ contains both $v_{4}$ and $v_{10}$. We have also developed a caching policy for shortest paths. However, we have not studied concise shortest paths. As we will detail in Section 4.3, we propose the notion of a generic concise shortest path that enables a trade-off between its size and the number of queries it can answer. This characteristic enables new caching techniques that require balancing between the lengths of paths and the hit ratios of paths.

\section{PROBLEM SETTING}

We first provide definitions for our problem, and then we introduce our objectives. Table 1 provides an overview of the notations used throughout the paper.

\begin{tabular}{|c|c|}
\hline Symbol & Meaning \\
\hline$G(V, E, W)$ & a graph \\
\hline$v_{i}$ & a node in the node set $V$ \\
\hline$\left(v_{i}, v_{j}\right)$ & an edge in the edge set $E$ \\
\hline$Q_{s, t}$ & a shortest path query from node $v_{s}$ to node $v_{t}$ \\
\hline$S P_{s, t}$ & a shortest path result of $Q_{s}, t$ \\
\hline$\left|S P_{s, t}\right|$ & the size of $S P_{s, t}$ (in number of nodes) \\
\hline$C P$ & a concise shortest path \\
\hline$G C P$ & a generic concise shortest path \\
\hline$\Psi$ & the cache \\
\hline
\end{tabular}

Table 1: Table of symbols

DEFINITION 1 (GRAPH). Let $G(V, E, W)$ be a directed spatial graph with a set $V$ of nodes and a set $E \subseteq V \times V$ of edges. Each node $v_{i} \in V$ models a road junction and has lat-long coordinates. Each edge $\left(v_{i}, v_{j}\right) \in E$ models a road segment, and $W: E \rightarrow R$ assigns a positive weight to each edge. 
Definition 2 (QUeRY AND Result). A shortest path query, denoted by $Q_{s, t}$, consists of a source and a target node, $v_{s}$ and $v_{t}$. The result of $Q_{s, t}$, denoted by $S P_{s, t}$, is a sequence of nodes from $v_{s}$ to $v_{t}$ such that it has the smallest sum of edge weights (among all paths from $v_{s}$ to $v_{t}$ in $G$ ).

By the optimal subpath property [8], any subpath of a shortest path $S P$ is also a shortest path. Thus, a query $Q_{s, t}$ can be answered by $S P$ if $v_{s}, v_{t} \in S P$ and $v_{s}$ appears before $v_{t}$ in $S P$. We denote this event by: $Q_{s, t} \subset S P$. We proceed to define the concept of cache hit.

DEFINITION 3 (CACHE HIT). Let a cache $\Psi$ be a set of shortest paths. A query $Q_{s, t}$ obtains a hit from $\Psi$ if there exists some $S P \in \Psi$ such that $v_{s}, v_{t} \in S P$ and $v_{s}$ appears before $v_{t}$ in $S P$. We denote this event by: $Q_{s, t} \subset \Psi$.

DEFINITION 4 (CACHE SIZE). Let $|P|$ denote the size (number of nodes) of a path. The total size of the cache is defined as: $\|\Psi\|=\sum_{P \in \Psi}|P|$.

We illustrate the above definitions with the example in Figure 1. Suppose that a cache contains two shortest paths: $\Psi=$ $\left\{S P_{1,10}, S P_{2,6}\right\}$, where $S P_{1,10}=\left\langle v_{1}, v_{3}, v_{4}, v_{5}, v_{7}, v_{9}, v_{10}\right\rangle$ and $S P_{2,6}=\left\langle v_{2}, v_{5}, v_{6}\right\rangle$. The cache size is: $\|\Psi\|=\left|S P_{1,10}\right|+$ $\left|S P_{2,6}\right|=7+3=10$. Consider the queries $Q_{3,7}$ and $Q_{1,2}$. Query $Q_{3,7}$ obtains a cache hit because the cached path $S P_{1,10}$ contains both query nodes $v_{3}$ and $v_{7}$. On the other hand, the query $Q_{1,2}$ does not obtain a cache hit.

We adopt the standard client-server architecture shown in Figure 3 as the setting. A cache server is placed in-between the mobile clients and an online shortest path service (e.g., Google Directions [1]). Upon receiving a shortest path query $Q_{s, t}$, the cache server checks whether there is a cache hit. If yes then it returns the result. Otherwise, it forwards $Q_{s, t}$ to the online shortest path service and eventually returns the result path $P_{s, t}$ computed by the service.

We assume that the dominant cost is that of the network traffic between the cache server and the shortest path service. Thus, our objective is to maximize the hit ratio of the cache server (subject to a given cache capacity) $[16,17,19]$. While the shortest path service may deploy a shortest path index $[4,11,20,26]$ for its own performance reasons, the introduction of such an index does not improve the cache hit ratio and is orthogonal to our work.

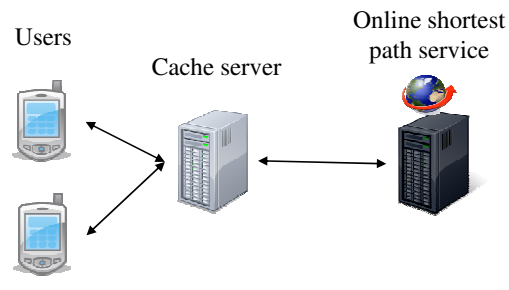

Figure 3: Client server architecture

Unlike previous work [22], this paper utilizes concise shortest paths to improve the hit ratio of caching. We elaborate on concise shortest paths and their operations in Section 4, and we present caching methods for them in Sections 5 and 6.

As a remark, two issues are orthogonal to this paper. First, we reuse existing data structures for the caching of shortest paths [22] to reduce the CPU overhead for maintaining cache structures. Second, we assume that the edge weights $W\left(v_{i}, v_{j}\right)$ maintained at the online shortest path service are independent of time. This is the case for edge weights that model travel distances (e.g., in MapQuest). If this is not the case, we may associate each cache item (shortest path) with a expiry time [9].

\section{CONCISE SHORTEST PATHS}

For navigation purposes, it suffices for mobile users to know driving instructions (e.g., going straight, making turns) instead of complete shortest paths. As discussed in the introduction, the notion of a concise shortest path is equivalent to driving instructions.

We first provide definitions for concise shortest paths. Then, we propose a server-side algorithm for converting a shortest path into a concise shortest path. Also, we present a client-side algorithm that enables a user to navigate using a concise shortest path. Finally, we introduce generic concise shortest paths.

\subsection{Definition and Examples}

We consider three possible driving instructions for mobile users, as illustrated in Figure 4. Suppose that the user is reaching a current node $v_{c}$ from a previous node $v_{p}$. In each case in the figure, the shortest path is indicated by bold arrows.

- Case I: The driving instruction is 'continue' to the next node $v_{n}$ as this is the only option.

- Case II: There is more than one option (e.g., travel to $v_{n 1}$ or to $v_{n 2}$ ). These options can be distinguished by their deviation angles from the previous travel direction (see Definition 5). In this figure, the deviation angles of $\left(v_{c}, v_{n 1}\right)$ and $\left(v_{c}, v_{n 2}\right)$ are $\delta_{p, c, n 1}$ and $\delta_{p, c, n 2}$, respectively. If the correct choice is the one with the smallest deviation angle, like in this figure, the driving instruction is 'go straight, by the smallest angle'.

- Case III: The correct choice is not the option with the smallest deviation angle. In this case, we must provide an explicit driving instruction: 'turn clockwise/anticlockwise by $x^{o}$, where $\mathrm{X}^{o}$ is the deviation angle $\delta_{p, c, n}$ (see Definition 5).

The above driving instructions are not explicitly stored in a concise shortest path. In Section 4.2, we provide a client-side algorithm to reconstruct driving instructions from a concise shortest path, during navigation.

Definition 5 (DEViation ANGLE). Given two adjacent edges $\left(v_{p}, v_{c}\right)$ and $\left(v_{c}, v_{n}\right)$, the deviation angle between them is:

$$
\delta_{p, c, n}=\left|180^{\circ}-\measuredangle v_{p} v_{c} v_{n}\right|
$$

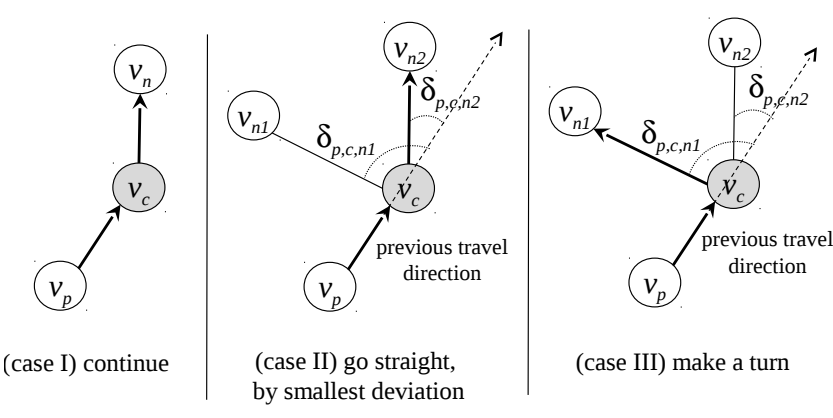

Figure 4: Possible driving instructions; the shortest path is indicated by bold arrows

Based on the above driving instructions, we define a concise shortest path as follows. 
DEFINITION 6 (CONCISE SHORTEST PATH). Let a shortest path $S P_{s, t}=\left\langle v_{\rho(1)}, v_{\rho(2)}, \cdots, v_{\rho(l)}\right\rangle$ be given, where $v_{\rho(i)}$ is the $i$-th node in $S P_{s, t}, s=\rho(1)$, and $t=\rho(l)$. A concise shortest path $C P_{s, t}$ is a subsequence of $S P_{s, t}$ such that: (i) $C P_{s, t}$ contains $v_{s}$ and $v_{t}$, and (ii) $C P_{s, t}$ contains $v_{\rho(i)}, v_{\rho(i+1)}$ if nodes $v_{\rho(i-1)}, v_{\rho(i)}$ and, $v_{\rho(i+1)}$ satisfy case III.

We illustrate a concise shortest path in Figure 5. Let the source and target nodes be $v_{1}$ and $v_{11}$, respectively. The shortest path $S P_{1,11}=\left\langle v_{1}, v_{2}, v_{4}, v_{5}, v_{7}, v_{8}, v_{9}, v_{11}\right\rangle$ is shown with bold edges. According to Definition 6, the concise shortest path is: $C P_{1,11}=$ $\left\langle v_{1}, v_{4}, v_{5}, v_{11}\right\rangle$. First, it must contain both the source and target nodes $\left(v_{1}\right.$ and $\left.v_{11}\right)$. Since $\left\langle v_{2}, \mathbf{v}_{4}, v_{5}\right\rangle$ matches case III, $v_{4}$ and the next node $\left(v_{5}\right)$ are in $C P_{1,11}$. The remaining nodes either match case I (e.g., $\left.v_{7}, v_{8}\right)$ or case II (e.g., $\left.v_{2}, v_{9}\right)$, so they are not in $C P_{1,11}$. Observe that a concise path describes the driving instructions for $S P_{s, t}$ unambiguously. Directions are given exactly when it is necessary to change the travel direction.

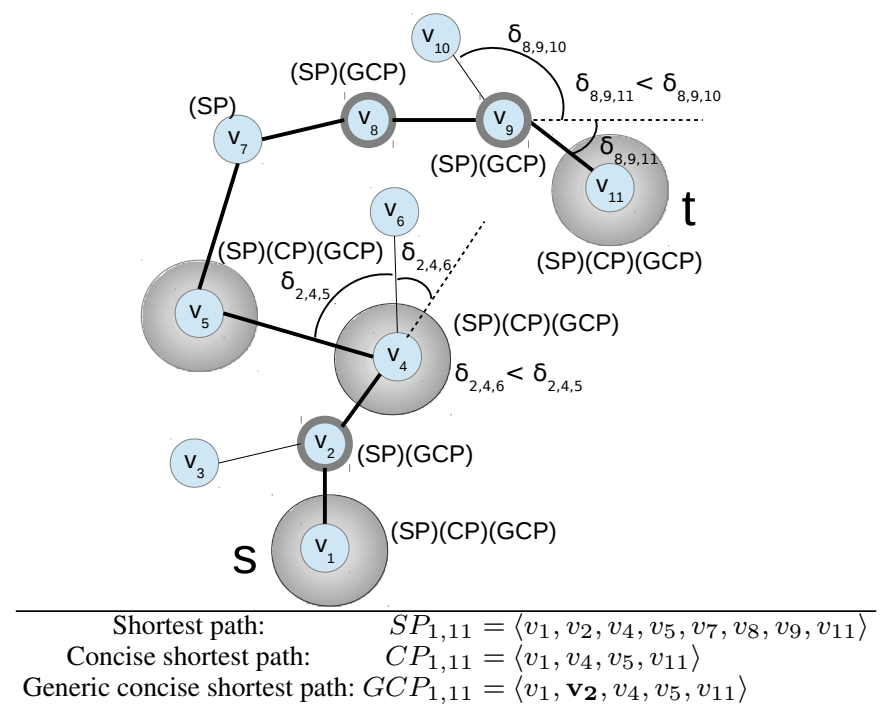

Figure 5: Concise shortest path

Concise shortest paths have the advantage that they consume less space while still offering sufficient information for driving.

\subsection{Operations}

We study caching methods for concise shortest paths in Sections 5 and 6 . Given a shortest path $S P$, the cache server can execute Algorithm 1 to extract a concise shortest path from $S P$.

First, we specify the initial travel direction (in Lines 3-6). We add the two first nodes of $S P$ to $C P$ if the first node has multiple branches, i.e., $\operatorname{deg}\left(v_{\rho(1)}\right)>1$. Otherwise, there is only one option so it suffices to add the first node of $S P$ to $C P$.

For each subsequent node $v_{\rho(i)}$ on $S P$, we compute the deviation angle to each adjacent node and then take the smallest deviation angle as $\delta^{*}$. If the next node $v_{\rho(i+1)}$ on $S P$ does not have the smallest deviation angle, we specify the travel direction by adding the current and the next nodes $\left(v_{\rho(i)}, v_{\rho(i+1)}\right)$ of $S P$ to $S P$. Finally, we add the last node of $S P$ to $C P$.

Upon receiving a concise shortest path $C P$ from the cache server, the client can apply Algorithm 2 to navigate along $C P$. Note that this algorithm also works correctly for extended versions of $C P$, which we discuss below.
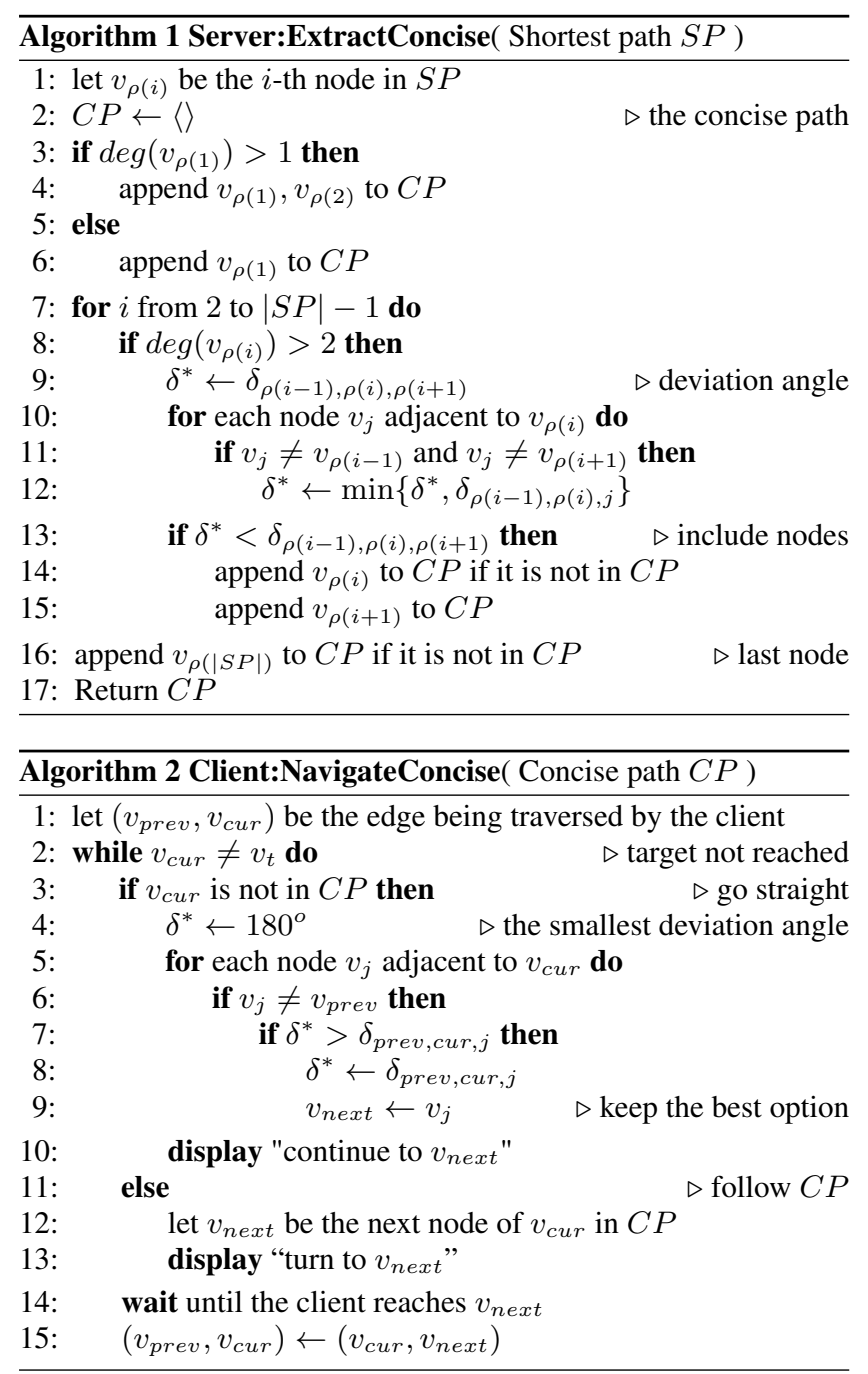

\subsection{Generic Concise Shortest Paths}

Although a concise shortest path occupies less space than a corresponding (complete) shortest path, it covers fewer nodes and thus it may answer a smaller number of queries. For example, suppose that the query $Q_{2,11}$ is a frequently-used query in Figure 5. Observe that the shortest path $S P_{1,11}$ can answer $Q_{2,11}$, while the concise path $C P_{1,11}$ cannot do so as it does not contain $v_{2}$.

To address this issue, we consider generic versions of concise shortest paths that include additional nodes:

DEFINITION 7 (GENERIC CONCISE SHORTEST PATH). A sequence $G C P_{s, t}$ is said to be a generic concise shortest path (from node $v_{s}$ to node $v_{t}$ ) if it is a subsequence of $S P_{s, t}$ and a supersequence of $C P_{s, t}$.

As an example, consider the map in Figure 5. Table 2 illustrates all generic concise shortest paths from source $v_{1}$ to target $v_{11}$. Note that the number of generic concise shortest paths is $2^{\left|S P_{s, t}\right|-\left|C P_{s, t}\right|}=2^{8-4}=16$. In the next section, we discuss how to select a generic concise shortest path in order to maximize the cache hit ratio.

\section{STATIC CACHING SETTING}

Static caching focuses on caching the most popular data items [5, $16,18]$. It utilizes a query $\log$ that records past queries in order 


\begin{tabular}{c|c}
$C P_{1,11}$ & $\left\langle v_{1}, v_{4}, v_{5}, v_{11}\right\rangle$ \\
\hline \multirow{3}{*}{ other } & $\left\langle v_{1}, v_{2}, v_{4}, v_{5}, v_{11}\right\rangle\left\langle v_{1}, v_{4}, v_{5}, v_{7}, v_{11}\right\rangle$ \\
generic & $\left\langle v_{1}, v_{4}, v_{5}, v_{8}, v_{11}\right\rangle\left\langle v_{1}, v_{4}, v_{5}, v_{9}, v_{11}\right\rangle$ \\
concise & $\left\langle v_{1}, v_{2}, v_{4}, v_{5}, v_{7}, v_{11}\right\rangle\left\langle v_{1}, v_{2}, v_{4}, v_{5}, v_{8}, v_{11}\right\rangle$ \\
paths & $\left\langle v_{1}, v_{2}, v_{4}, v_{5}, v_{9}, v_{11}\right\rangle\left\langle v_{1}, v_{4}, v_{5}, v_{7}, v_{8}, v_{11}\right\rangle$ \\
& $\left\langle v_{1}, v_{4}, v_{5}, v_{7}, v_{9}, v_{11}\right\rangle\left\langle v_{1}, v_{4}, v_{5}, v_{8}, v_{9}, v_{11}\right\rangle$ \\
& $\left\langle v_{1}, v_{2}, v_{4}, v_{5}, v_{7}, v_{8}, v_{11}\right\rangle\left\langle v_{1}, v_{2}, v_{4}, v_{5}, v_{7}, v_{9}, v_{11}\right\rangle$ \\
& $\left\langle v_{1}, v_{2}, v_{4}, v_{5}, v_{8}, v_{9}, v_{11}\right\rangle\left\langle v_{1}, v_{4}, v_{5}, v_{7}, v_{8}, v_{9}, v_{11}\right\rangle$ \\
\hline$S P_{1,11}$ & $\left\langle v_{1}, v_{2}, v_{4}, v_{5}, v_{7}, v_{8}, v_{9}, v_{11}\right\rangle$
\end{tabular}

Table 2: Generic concise shortest paths (from $v_{1}$ to $v_{11}$ )

to determine the access frequencies of data items. Static caching incurs low overhead at runtime; however, it may not adapt well to a varying query distribution.

We present a caching method for generic concise shortest paths that applies an existing static caching policy for shortest paths [22]. The frequencies of queries in the query log are used to define a 'benefit' score that captures the 'importance' of a path in answering queries. Our main challenge is to find generic concise paths that have high 'benefit' scores.

In Section 5.1, we give a benefit score model for generic concise paths. Then, in Section 5.2, we present a method for computing a generic concise path with a high benefit score. Finally, we propose an efficient implementation in Section 5.3.

\subsection{Benefit Model}

We adopt the benefit model presented in previous work [22] to quantify the importance of a path in answering queries. First, we provide definitions of query $\log$ and query frequency.

\section{DEFINITION 8 (QUERY LOG AND FREQUENCY).}

A query $\log \mathcal{Q} \mathcal{L}$ is a collection of shortest path queries that have been issued by users in the past.

The query frequency $\chi_{s, t}$ of a query $Q_{s, t}$ is defined as the number of occurrences of $Q_{s, t}$ in the query $\log \mathcal{Q} \mathcal{L}$.

$$
\chi_{s, t}=\left|\left\{Q_{s, t} \in \mathcal{Q L}\right\}\right|
$$

Table 3 illustrates the values of $\chi_{s, t}$ derived from an example query $\log$ for the map in Figure 5. For simplicity, since we assume $\chi_{s, t}=\chi_{t, s}$.

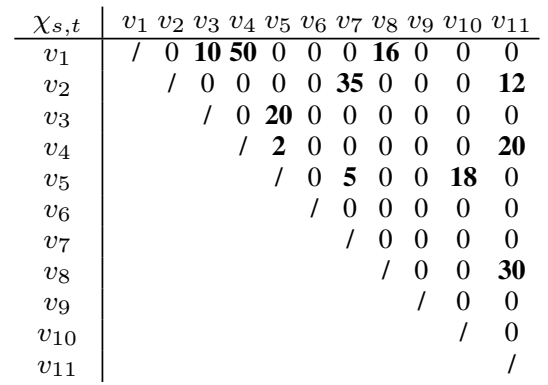

Table 3: Example of $\chi_{s, t}$ values for the graph, with $\chi_{s, t}=\chi_{t, s}$

Given a generic concise shortest path $P$, we define its benefit $\Upsilon(P)$ as:

$$
\Upsilon(P)=\sum_{v_{i}, v_{j} \in P, Q_{i, j} \subset P} \chi_{i, j}
$$

The benefit is the number of queries (in $\mathcal{Q} \mathcal{L}$ ) that can be answered by $P$. As a remark, a simple (nested-loop) implementation for computing $\Upsilon(P)$ would take $O\left(|P|^{2}\right)$ time.
Then, we define the benefit of a cache $\Psi$ as follows:

$$
\Upsilon(\Psi)=\sum_{v_{i}, v_{j} \in V, Q_{i, j} \subset \Psi} \chi_{i, j}
$$

As an example, consider the cache content in Table 4 and the map in Figure 5. The path $C P_{1,11}$ has benefit: $\Upsilon\left(C P_{1,11}\right)=\chi_{1,4}+$ $\chi_{1,5}+\chi_{1,11}+\chi_{4,5}+\chi_{4,11}+\chi_{5,11}=50+0+0+2+20+0=72$. The benefit of the cache $\Psi$ is: $\Upsilon(\Psi)=\chi_{1,4}+\chi_{4,5}+\chi_{4,11}+\chi_{3,5}=$ $50+2+20+20=92$. Observe that Equation 4 avoids duplicate counting. For example, the term $\chi_{4,5}$ appears only once in the equation of $\Upsilon(\Psi)$, despite that the query $Q_{4,5}$ can be answered by paths $C P_{1,11}, C P_{3,8}$, and $C P_{4,9}$.

\begin{tabular}{c|c} 
& concise path $C P$ \\
\hline$C P_{1,11}$ & $\left\langle v_{1}, v_{4}, v_{5}, v_{11}\right\rangle$ \\
$C P_{3,8}$ & $\left\langle v_{3}, v_{4}, v_{5}, v_{8}\right\rangle$ \\
$C P_{4,9}$ & $\left\langle v_{4}, v_{5}, v_{9}\right\rangle$
\end{tabular}

Table 4: A cache $\Psi$ of concise shortest paths

With the above benefit model, we formulate our static caching problem as follows:

Problem 1 (Static CACHING Problem). Select a set of generic concise shortest paths $\Psi=\left\{P_{s, t}\right\}$ such that: (i) the benefit $\Upsilon(\Psi)$ is maximized, and (ii) the cache size $\|\Psi\|$ is bounded by a given cache capacity value.

In previous work [22], we require each $P_{s, t}$ to be a shortest path. Here, we consider a much larger search space and allow each $P_{s, t}$ to be a generic concise shortest path.

\subsection{Benefit-Based Generic Concise Path}

Given a shortest path $S P_{s, t}$, we want to construct a generic concise path $G C P_{s, t}$ such that it has a high benefit and a small size.

Given the paths present in the cache $\Psi$, we define the marginal benefit of a path $P$ with respect to $\Psi$ as:

$$
\begin{aligned}
\Upsilon_{m}(P \backslash \Psi) & =\Upsilon(\Psi \cup\{P\})-\Upsilon(\Psi) \\
& =\sum_{v_{i}, v_{j} \in P, Q_{i, j} \in P, Q_{i, j} \not \subset \Psi} \chi_{i, j}
\end{aligned}
$$

This notion captures the total frequencies of queries (in $\mathcal{Q} \mathcal{L}$ ) that can be answered by $P$ but not by paths in $\Psi$. Intuitively, it is desirable to cache a path $P$ if the benefit $\Upsilon_{m}(P \backslash \Psi)$ is high and the size $|P|$ is small. Thus, we define the normalized benefit of a path $P$ as: $\overline{\Upsilon_{m}}(P \backslash \Psi)=\frac{\Upsilon_{m}(P \backslash \Psi)}{|P|}$.

A brute-force approach is to enumerate all possible generic concise shortest paths (like in Table 2) and then find the one with the highest $\overline{\Upsilon_{m}}$ value. However, as discussed in Section 4.3, the number of possible generic concise shortest paths is exponential in the path size.

In the following, we present a greedy heuristic solution to solve this problem in polynomial time. Algorithm 3 is the pseudo-code of this solution. It takes a shortest path $S P_{s, t}$ and its corresponding concise path $C P_{s, t}$ as input. We denote $B P$ as the path with the highest $\bar{\Upsilon}_{m}$ value found so far. It is initialized to $C P_{s, t}$. The set $S$ contains the possible nodes that can be added to $B P$. In each iteration, we compute the benefit of the path $B P \cup\left\{v_{c}\right\}$ for each $v_{c}$ (Lines 5-9), and find the node (say $v_{\text {best }}$ ) with the highest benefit. If the normalized benefit of $B P \cup\left\{v_{\text {best }}\right\}$ is higher than that of $B P$, we add $v_{\text {best }}$ to $B P$ and repeat the loop. Otherwise, the algorithm terminates.

We illustrate the working of algorithm 3 in Table 5. Here, we are given a shortest path $S P_{1,11}=\left\langle v_{1}, v_{2}, v_{4}, v_{5}, v_{7}, v_{8}, v_{9}, v_{11}\right\rangle$ and 


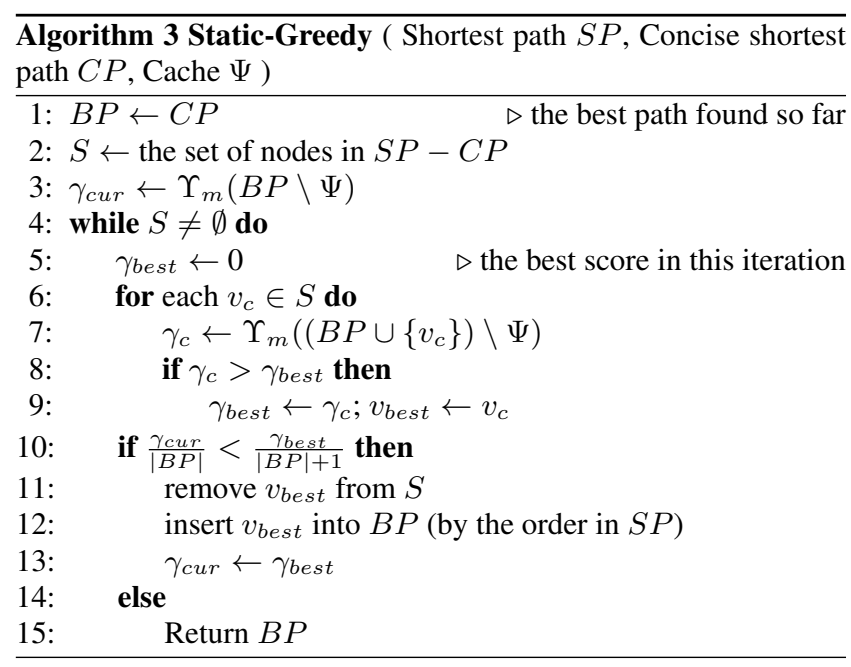

its corresponding concise shortest path $C P_{1,11}=\left\langle v_{1}, v_{4}, v_{5}, v_{11}\right\rangle$. Note that Table 5a shows only the rows and columns whose nodes fall into path $S P_{1,11}$. Table $5 \mathrm{~b}$, shows the running steps of the algorithm. First, we initialize $B P$ to $C P_{1,11}$ and the set $S$ to $\left\{v_{2}, v_{7}, v_{8}, v_{9}\right\}$. Those $\chi_{s, t}$ entries that contribute to the benefit of $B P$ are shaded light-gray (see Table 5a). In iteration 1 , we add the best node $v_{8}$ to $B P$ because its normalized benefit is higher than that of $B P$. Those $\chi_{s, t}$ entries that contribute to the additional benefit of $B P$ are shaded medium-gray. In iteration 2, even for the best node $v_{2}$, the normalized benefit of $B P \cup\left\{v_{2}\right\}$ is smaller than that of $B P$. Thus, the algorithm terminates and returns the path $\left\langle v_{1}, v_{4}, v_{5}, v_{8}, v_{11}\right\rangle$.

\begin{tabular}{c|cccccccc}
$\chi_{s, t}$ & $v_{1}$ & $v_{2}$ & $v_{4}$ & $v_{5}$ & $v_{7}$ & $v_{8}$ & $v_{9}$ & $v_{11}$ \\
\hline$v_{1}$ & $/$ & 0 & 50 & 0 & 0 & 16 & 0 & 0 \\
$v_{2}$ & & $/$ & 0 & 0 & 35 & 0 & 0 & 12 \\
$v_{4}$ & & & $/$ & 2 & 0 & 0 & 0 & 20 \\
$v_{5}$ & & & & $/$ & 5 & 0 & 0 & 0 \\
$v_{7}$ & & & & & $/$ & 0 & 0 & 0 \\
$v_{8}$ & & & & & & $/$ & 0 & 30 \\
$v_{9}$ & & & & & & & $/$ & 0 \\
$v_{11}$ & & & & & & & & $/$
\end{tabular}

(a) relevant entries of $\chi_{s, t}$

\begin{tabular}{c|c|c} 
Iteration & Steps & Path \\
\hline Initialization & $C P=\left\langle v_{1}, v_{4}, v_{5}, v_{11}\right\rangle$ & $\left\langle v_{1}, v_{4}, v_{5}, v_{11}\right\rangle$ \\
& $S=\left\{v_{2}, v_{7}, v_{8}, v_{9}\right\}$ & $\bar{\Upsilon}_{m}=72 / 4=\mathbf{1 8}$ \\
\hline$(1)$ & $v_{2}:(72+12) / 5$ & \\
& $v_{7}:(72+5) / 5$ & \\
$v_{8}:(72+16+30) / 5$ & \\
& $v_{9}:(72+0) / 5$ & Add $v_{8}$ \\
& Highest: $23.6>18$ & $\left\langle v_{1}, v_{4}, v_{5}, v_{8}, v_{11}\right\rangle$ \\
& & $\Upsilon_{m}=118 / 5=\mathbf{2 3 . 6}$ \\
\hline$(2)$ & $v_{2}:(118+12) / 6$ & \\
& $v_{7}:(118+5) / 6$ & STOP \\
& $v_{9}:(118+0) / 6$ & \\
& Highest: $21.67<23.6$ & \\
& (b) running steps & $\left\langle v_{1}, v_{4}, v_{5}, v_{8}, v_{11}\right\rangle$
\end{tabular}

(b) running steps

Table 5: Finding the best generic concise shortest path, for $S P_{1,11}=\left\langle v_{1}, v_{2}, v_{4}, v_{5}, v_{7}, v_{8}, v_{9}, v_{11}\right\rangle$ and $C P_{1,11}=\left\langle v_{1}, v_{4}, v_{5}, v_{11}\right\rangle$

As a remark, the above algorithm may not always return the optimal result (i.e., the generic concise path with the highest normalized benefit). Suppose that we add the nodes $v_{2}, v_{7}, v_{8}$ to $C P_{1,11}$ to form a generic concise path: $P^{*}=\left\langle v_{1}, v_{2}, v_{4}, v_{5}, v_{7}, v_{8}, v_{11}\right\rangle$. According to Table 5a, the normalized benefit of $P^{*}$ is: $(118+$ $35+12+5) / 7=24.28$, which is higher than the algorithm's result (23.6).

\section{Time Complexity Analysis.}

Let $n$ be the number of nodes in $S P$. Note that both the sizes of $S$ and $B P$ are upper-bounded by $n$.

In the while-loop (Lines 4-15), we remove a node $v_{\text {best }}$ from $S$ in each iteration, so it has at most $n$ iterations. The for loop (Lines 6-9) has at most $n$ iterations because $S$ contains at most $n$ nodes. In Line 7, each call to $\Upsilon_{m}\left(\left(B P \cup\left\{v_{c}\right\}\right) \backslash \Psi\right)$ takes $O\left(n^{2}\right)$ time as the path size is at most $n$. By combining the above, the total running time of the while-loop is $O\left(n^{4}\right)$.

Before the while-loop, Lines 1-2 take $O(n)$ time and Line 3 takes $O\left(n^{2}\right)$ time. Thus, the time complexity of the algorithm is $O\left(n^{4}\right)$.

The computational cost is high, even though the typical path size $n$ is in the order of hundreds on real road networks.

\subsection{Efficient Implementation}

We proceed to present a more efficient implementation of Algorithm 3 . The idea is to identify shared expressions in the calculation and compute such expressions only once, regardless of the path size $n(n=|S P|)$.

Recall that it takes $O\left(n^{2}\right)$ time to compute $\Upsilon_{m}(P \backslash \Psi)$, where $P$ is a subsequence of $S P$. Consider the scenario that we need the updated $\gamma$ value after adding a node $v_{c}$ into $P$. Fortunately, we can apply Equation 6 to derive $\Upsilon_{m}\left(\left(P \cup\left\{v_{c}\right\}\right) \backslash \Psi\right)$ from $\Upsilon_{m}(P \backslash \Psi)$ incrementally. This derivation requires only $O(n)$ time to compute $\sum_{v_{j} \in P, Q_{c, j} \not \subset \Psi} \chi_{c, j}$ because we have one node for $v_{c}$ and at most $n$ nodes in $P$.

$$
\begin{aligned}
& \Upsilon_{m}\left(\left(P \cup\left\{v_{c}\right\}\right) \backslash \Psi\right) \\
& =\sum_{v_{i}, v_{j} \in P \cup\left\{v_{c}\right\}, Q_{i, j} \not \subset \Psi} \chi_{i, j} \\
& =\sum_{v_{i} \in P \cup\left\{v_{c}\right\}} \sum_{v_{j} \in P \cup\left\{v_{c}\right\}, Q_{i, j} \not \subset \Psi} \chi_{i, j} \\
& =\left(\sum_{v_{i} \in P} \sum_{v_{j} \in P, Q_{i, j} \not \subset \Psi}+\sum_{v_{i} \in P, Q_{i, c} \not \subset \Psi}+\sum_{v_{j} \in P, Q_{c, j} \not \subset \Psi}\right) \chi_{i, j} \\
& =\Upsilon_{m}(P \backslash \Psi)+2 \cdot \sum_{v_{j} \in P, Q_{c, j} \not \subset \Psi} \chi_{c, j}
\end{aligned}
$$

We propose an efficient implementation in Algorithm 4. For each node $v_{c}$ in $S$, we maintain its benefit $\Upsilon_{m}\left(\left(P \cup\left\{v_{c}\right\}\right) \backslash \Psi\right)$ in an attribute $v_{c} \cdot \gamma$. We first compute $\Upsilon_{m}(B P \backslash \Psi)$ at Line 3, then derive $v_{c} \cdot \gamma$ for each node $v_{c} \in S$ incrementally.

The while-loop (Lines 6-21) repeats until $S$ becomes empty or the benefit of $B P$ cannot be improved further. In each iteration, we simply find the node ( $\left.v_{\text {best }}\right)$ with the highest $\gamma$. If the normalized benefit $\overline{\Upsilon_{m}}$ of $B P \cup\left\{v_{\text {best }}\right\}$ is better than that of $B P$, we insert $v_{\text {best }}$ into $B P$. Also, we need to update the value $v_{c} \cdot \gamma$ for each remaining node $v_{c} \in S$ (Lines 13-17). By using the idea in Equation 6, we can derive a shared expression for updates (Line 13). We can compute it once before using it to update each $v_{c} \cdot \gamma$.

\section{Time Complexity Analysis.}

We proceed as in the time complexity analysis in Section 5.2. Let $n$ be the number of nodes in $S P$. Again, both the sizes of $S$ and $B P$ are upper-bounded by $n$. 


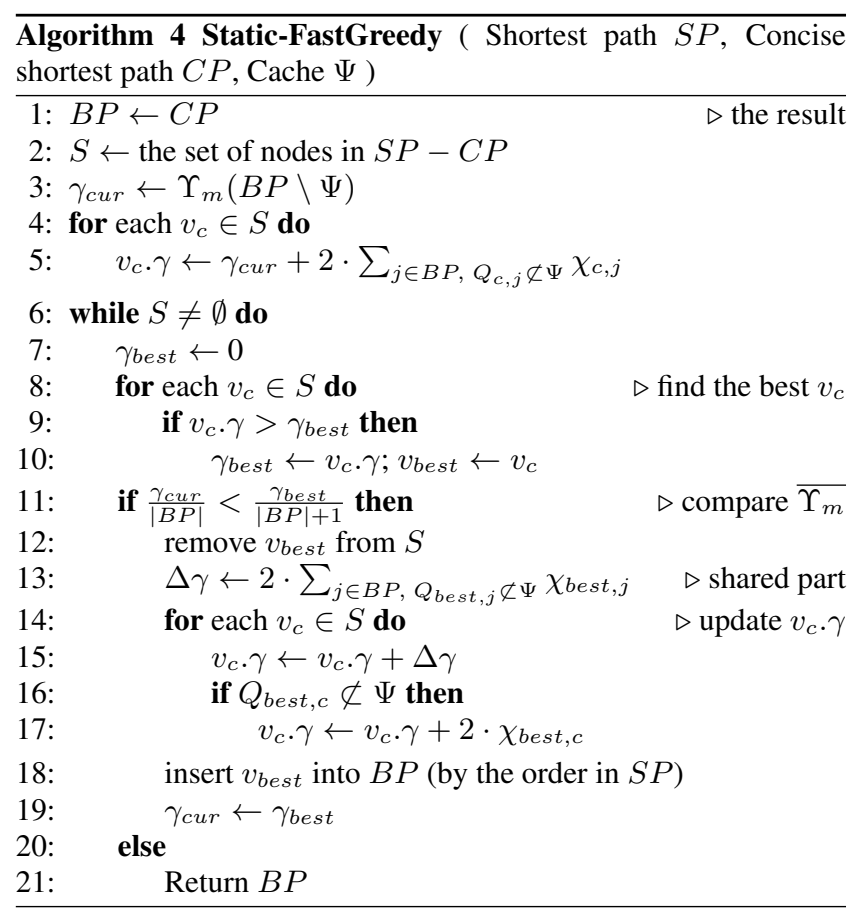

The while-loop (Lines 6-21) has at most $n$ iterations. Within the while-loop, the first for loop (Lines 8-10) takes $O(n)$ time, the summation (Line 13) takes $O(n)$ time, and the second for loop (Lines 14-17) takes $O(n)$ time. Thus, the time complexity of the while-loop is $O\left(n^{2}\right)$.

Before the while-loop, Lines 1-2 take $O(n)$ time, and Lines 34 take $O\left(n^{2}\right)$ time. Thus, the time complexity of the algorithm is $O\left(n^{2}\right)$.

\section{DYNAMIC CACHING SETTING}

We have examined the static caching approach in Section 5. In this section, we adopt the dynamic caching $[10,15,16]$ approach, which intends to cache the most recently accessed data items. When a cache miss occurs and the cache is full, these policies decide which data item to evict from the cache. For instance, the Least-Recently-Used (LRU) policy evicts the least recently used item in the cache. These policies allow the cache to adapt dynamically to the query workload. However, they incur runtime overhead in maintaining the cache.

In this section, we present a dynamic caching method for generic concise shortest paths. It adopts the LRU policy. When we obtain a shortest path $S P$ (after a cache miss), our main problem is how to compute a short generic shortest path that will be able to answer many queries (in the future). To tackle this problem, we employ two lightweight data structures for maintaining query statistics: (i) a sliding window $\mathbb{W}$ that keeps the most recent $\mathbb{W}_{\text {size }}$ queries, and (ii) an array $\mu$ that records the frequencies of the query nodes in $\mathbb{W}$.

Algorithm 5 shows the pseudo code for our dynamic caching method. It is invoked when there is a cache miss for a query $Q_{s, t}$. First, we calculate the shortest path $S P$ for $Q_{s, t}$, and then compute a concise shortest path $G C P$ for it. Then, for each node $v_{i}$ in $S P$, we add it into the DGCP if $v_{i}$ is in $C P$ or its frequency in $\mu$ is nonzero. Next, we remove the oldest query from the sliding window $\mathbb{W}$ and insert the newest query into $\mathbb{W}$. Then, we update the frequency array $\mu$ accordingly. Finally, we apply the LRU policy to update the cache $\Psi$ with the generic concise shortest path.

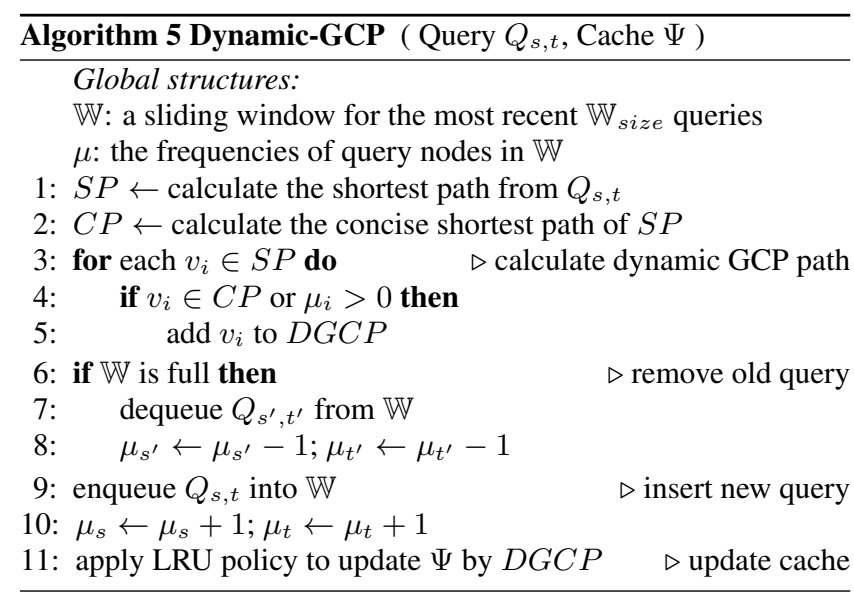

\begin{tabular}{cc} 
Query & Shortest Path \\
\hline$Q_{8,9}$ & $\left\langle v_{8}, v_{5}, v_{7}, v_{9}\right\rangle$ \\
$Q_{1,4}$ & $\left\langle v_{1}, v_{3}, v_{4}\right\rangle$ \\
$Q_{1,10}$ & $\left\langle v_{1}, v_{3}, v_{4}, v_{5}, v_{7}, v_{9}, v_{10}\right\rangle$ \\
$Q_{2,10}$ & $\left\langle v_{2}, v_{5}, v_{7}, v_{9}, v_{10}\right\rangle$
\end{tabular}

(a) shortest paths of queries

\begin{tabular}{l|cccc} 
Time $t$ & Query & $\Delta L$ & $\mu$ & DGCP Path \\
\hline \hline 0 & N.A. & N.A. & $1,3,5,6,7,10: \underline{1}$ & N.A. \\
\hline 1 & $(8,9)$ & $-(1,6)$ & $1,3,5,6,7,10: \underline{1}$ & $\left\langle v_{8}, v_{5}, \mathbf{v}_{\mathbf{7}}, v_{9}\right\rangle$ \\
& & $+(8,9)$ & & \\
\hline 2 & $(1,4)$ & $-(3,5)$ & $3,5,7,8,9,10: \underline{1}$ & $\left\langle v_{1}, \mathbf{v}_{\mathbf{3}}, v_{4}\right\rangle$ \\
& & $+(1,4)$ & & \\
\hline 3 & $(1,10)$ & $-(7,10)$ & $1,4,7,8,9,10: \underline{1}$ & $\left\langle v_{1}, v_{5}, v_{7}, \mathbf{v}_{\mathbf{9}}, v_{10}\right\rangle$ \\
& & $+(1,10)$ & & \\
\hline 4 & $(2,10)$ & $-(8,9)$ & $1: \underline{2} ; 4,8,9,10: \underline{1}$ & $\left\langle v_{2}, v_{5}, v_{7}, \mathbf{v}_{\mathbf{9}}, v_{10}\right\rangle$ \\
& & $+(2,10)$ & &
\end{tabular}

(b) running steps

Table 6: Running steps of dynamic caching, $L_{\text {size }}=3$, for the road network in Figure 1

We proceed to illustrate the running steps of the algorithm. Table $6 a$ shows a list of queries and their shortest paths. Table $6 \mathrm{~b}$ depicts the running steps. $\Delta L$ shows which query will be added and removed from $\mathbb{W}$ at each timestamp. Array $\mu$ shows the frequency of query nodes in the sliding window. In this example, the extra node(s) are added to the CP path (shown in bold) in order to obtain the DGCP path. At time $t=1$, we receive the query $(8,9)$ and calculate its DGCP path. Since calculation of DGCP paths happen before updating $\mu$, the content of $\mu$ does not change from the initial state. Then, we remove $(1,6)$ and add $(8,9)$ to $\mathbb{W}$. At time $t=2$, the query from $t=1$ affects the content of $\mu$. Window $\mathbb{W}$ is updated as before. Similarly, the running steps at $t=3$ and $t=4$ are shown in the table.

\section{EXPERIMENTAL STUDY}

Section 7.1 covers the methods considered in the experiments. Section 7.2 covers the experimental settings. Section 7.3 covers experiments on real trajectory induced workload, while Section 7.4 covers the experiments on synthetic workloads.

\subsection{Methods Considered}

We evaluate the hit ratios of our caching methods and competitors on a machine running Debian. Table 7 lists all the methods used in our experiments. In the static caching setting, all methods use an existing caching policy for shortest paths [22]. In the 
dynamic caching setting, all methods use LRU for cache replacement.

Our proposed methods are: (i) $\mathrm{CP}$, caching concise paths as defined in Section 4, (ii) GCP, the static caching method for generic concise paths in Section 5, and (iii) DGCP, the dynamic caching method for generic concise paths in Section 6.

As discussed in related work, K-Skip [21] is a lossy shortest path compression method, so we do not compare with it. We compare our methods with two competitors: (i) full shortest path (SP), and (ii) concise path skip (CP-Skip). CP-Skip is a variant of K-Skip that includes all nodes of CP and every $k$-th node of SP. Since CP-Skip contains $\mathrm{CP}$, it is a lossless shortest path method.

\begin{tabular}{c||cc} 
Method & Static caching policy & Dynamic caching policy \\
\hline \hline CP & {$[22]$} & LRU \\
\hline GCP & {$[22]$} & N.A. \\
\hline DGCP & N.A. & LRU \\
\hline SP & {$[22]$} & LRU \\
\hline CP-Skip & {$[22]$} & LRU
\end{tabular}

Table 7: Methods used in experiments

\subsection{Experimental Setting}

\section{Datasets and Workloads}

Table 8 shows information on the road networks used in the experiments. Although a real query log for online direction services [23] exist, service providers do not make their query logs publicly available. Thus, we generate query logs that each contains 300,000 queries.

We use real trajectories from the Aalborg [13] and Beijing [25] road networks. From each trajectory, we extract the start and end locations as the source and target nodes of a shortest path query in a query log. Since the trajectory datasets for Aalborg and Beijing are small ( $4.3 \mathrm{k}$ and $13 \mathrm{k}$ trajectories, respectively), we enlarge the trajectory dataset (to 300,000 trajectories) by sampling trajectories in the log and deviating their coordinates within a given radius (default value: $0.5 \mathrm{~km}$ )

For each of the two larger road networks (Colorado and New York), we generate a query log as follows. In the real world, drivers start from a dense region (e.g., a residential region) and travel towards another dense region (e.g., an industrial region). To simulate such behavior, we randomly choose a set of cluster centers with a given radius to form a set of clusters. Next, we randomly pick a pair of nodes from any 2 clusters to form a shortest path. By default, we use 10 clusters, and the radius is $8 \mathrm{~km}$.

Following an existing experimental methodology [22], we divide the query $\log$ into two equal parts: (i) a query $\log \mathcal{Q} \mathcal{L}$, for extracting query statistics and training the cache, and (ii) a query workload $\mathcal{W} \mathcal{L}$ used for measuring the hit ratios of the methods.

\begin{tabular}{l||cc} 
Road network & Map size $\left(\mathbf{k m}^{2}\right)$ & Road network \\
\hline \hline Aalborg & $60 \times 60$ & $\begin{array}{c}\text { download.cloudmade.com } \\
129 \mathrm{k} \text { nodes, } 137 \mathrm{k} \text { edges }\end{array}$ \\
\hline Beijing & $60 \times 60$ & $\begin{array}{c}\text { download.cloudmade.com } \\
76 \mathrm{k} \text { nodes, } 85 \mathrm{k} \text { edges }\end{array}$ \\
\hline Colorado & $1000 \times 1000$ & www.dis.uniroma1.it/challenge9 \\
& & 435k nodes, $1,057 \mathrm{k}$ edges \\
\hline New York & $6000 \times 4000$ & www.dis.uniroma1.it/challenge 9 \\
& & 264k nodes, $733 \mathrm{k}$ edges
\end{tabular}

Table 8: Characteristics of datasets

\section{Parameters and Default Values}

Table 9 lists the parameters and their default values. The first two entries show the default radiuses for the different road networks. Since different road networks have different sizes, we use $0.5 \mathrm{~km}$ as the default radius for the Aalborg and Beijing road networks, and we use $8 \mathrm{~km}$ as the default radius for the Colorado and New York road networks. The number of clusters denotes the number of clusters used for generating the synthetic workload (for Colorado and New York). The cache capacity (ratio) expresses the cache space as a percentage of the space needed for storing the entire road network. Thus, the absolute cache space for the different road networks are different. The last two are parameters for specific to CP-Skip and DGCP. Path size ratio is used by CP-Skip to define the ratio of the CP-Skip path length to the full shortest path length. Window size is used by DGCP to define the size of the sliding window.

\begin{tabular}{|l|l|}
\hline Parameter & Default value \\
\hline \hline Radius (for Aalborg, Beijing) & $0.5 \mathrm{~km}$ \\
\hline Radius (for Colorado, New York) & $8 \mathrm{~km}$ \\
\hline Number of clusters (for Colorado, New York) & 10 \\
\hline Cache capacity (ratio) & $50 \%$ \\
\hline Path size ratio (for CP-Skip) & $50 \%$ \\
\hline Window size (for DGCP) & 1000 queries \\
\hline
\end{tabular}

Table 9: Default parameters

\subsection{Real Trajectory Induced Workloads}

We proceed to report on experiments using real trajectory induced workloads (on the Aalborg and Beijing road networks).

First, we examine the average lengths (i.e., number of nodes) of paths in the cache for the different methods, see Table 10. Observe that full shortest paths (SP) are much longer than concise shortest paths (CP). Generic concise shortest paths (GCP/DGCP) are only slightly longer than concise shortest paths $(\mathrm{CP})$. This means that SP paths contain many intermediate nodes that do not intersect query nodes in the workload. Note that the average path lengths of the methods (e.g., SP and CP) are similar in the static and dynamic settings.

\begin{tabular}{|c||c|c|c|c|c|r|}
\hline \multicolumn{1}{|c||}{$\begin{array}{c}\text { Road } \\
\text { Network }\end{array}$} & \multicolumn{3}{c|}{ Static } & \multicolumn{3}{c|}{ Dynamic } \\
\cline { 2 - 7 } & SP & CP & GCP & SP & CP & DGCP \\
\hline \hline Aalborg & 338.5 & 38.7 & 48.1 & 315.6 & 37.3 & 41.3 \\
\hline Beijing & 166.1 & 26.4 & 35.6 & 129.8 & 23.1 & 26.1 \\
\hline Colorado & 874.8 & 98.3 & 115.7 & 887.3 & 96.9 & 106.1 \\
\hline New York & 501.9 & 71.3 & 73.3 & 514.7 & 70.4 & 72.0 \\
\hline
\end{tabular}

Table 10: Average path length (in nodes)

Next, we study the effect of the path size ratio on the performance of CP-Skip, see Figure 6. The other methods (SP, CP, GCP) are included for reference only; they do not take the path size ratio as a parameter. Clearly, GCP outperforms CP-Skip in all cases. The results for dynamic caching are similar, so we omit them.

Next, we investigate the effect of the window size parameter on the hit ratio of the DGCP method (in the dynamic caching setting). Figure 7 plots the hit ratio of DGCP with respect to the window size. The hit ratio increases until the window size reaches 10,000 . Observe that we can achieve a high hit ratio when the window size is sufficiently large.

Figure 8 shows the hit ratios of the methods as a function of the cache capacity, Again, GCP outperforms the other methods significantly, in both the static and dynamic settings. Since CP-Skip and 
Static

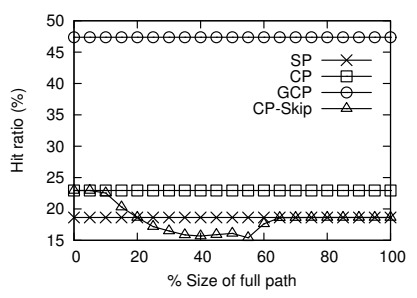

(a) Aalborg data

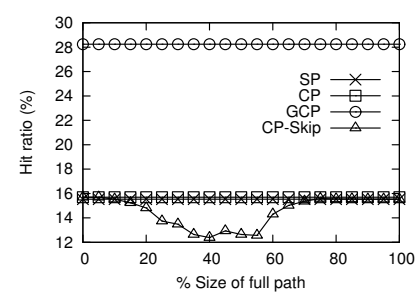

(b) Beijing data
Figure 6: Hit ratio vs. path size ratio

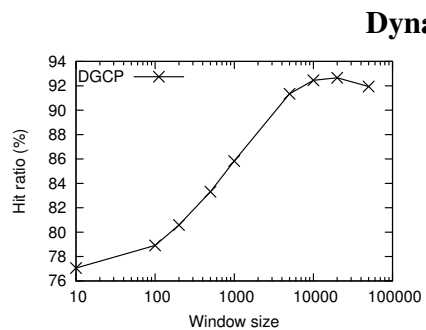

(a) Aalborg data
Dynamic

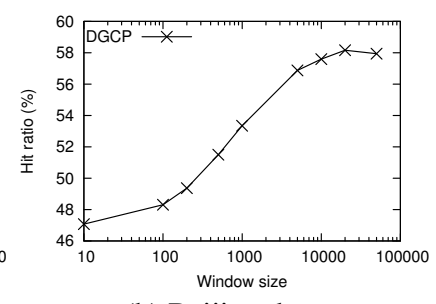

(b) Beijing data
Figure 7: Hit ratio vs. window size

SP have similar hit ratios, we exclude CP-Skip from subsequent experiments.

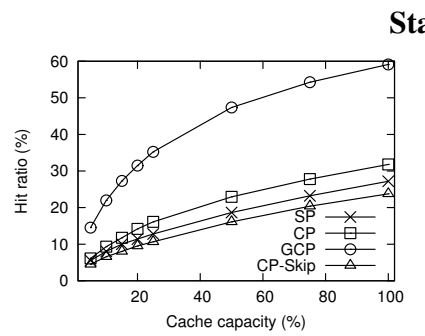

Static

Dynamic

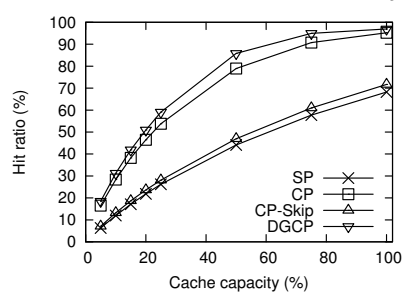

(a) Aalborg
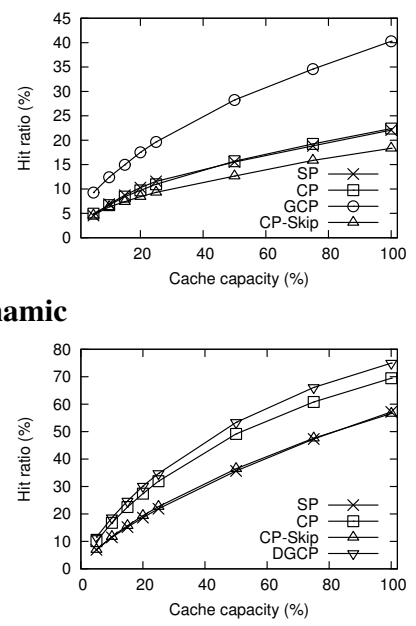

(b) Beijing data
Figure 8: Hit ratio vs. cache capacity

Figure 9 shows the hit ratios of the methods for various cluster radius values. Observe that a smaller radius leads to a higher hit ratio. This happens because smaller clusters result in fewer possible unique queries.

\subsection{Synthetic Workload}

We proceed to report on experiments using synethetic workload (on the Colorado and New York road networks).

We plot the average path length of the methods, as shown in Table 10. In general, the results are similar to those for the real trajectory induced workloads.

Figure 10 shows the hit ratios of the methods with respect to the
Static
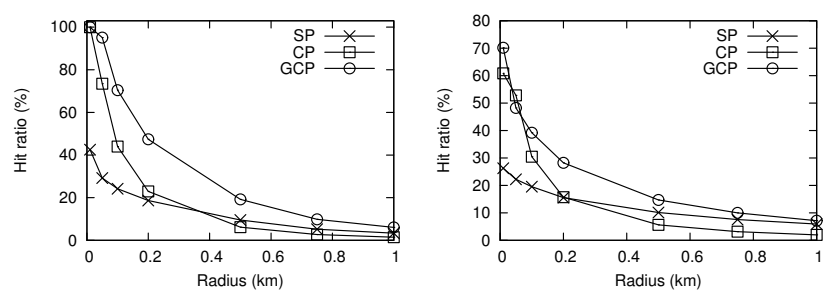

Dynamic

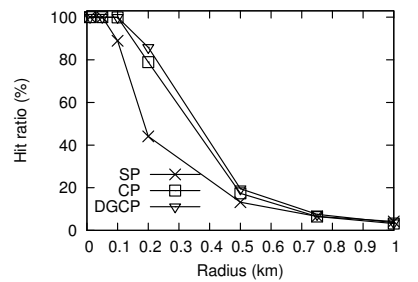

(a) Aalborg

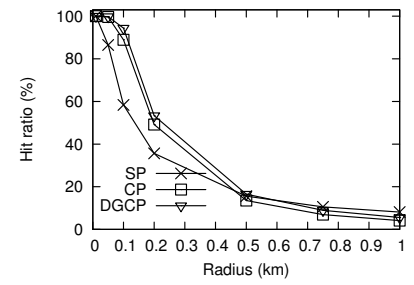

(b) Beijing
Figure 9: Hit ratio vs. cluster radius

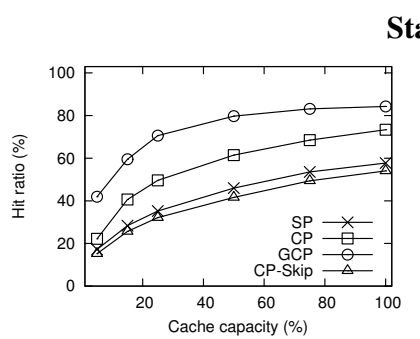

Static

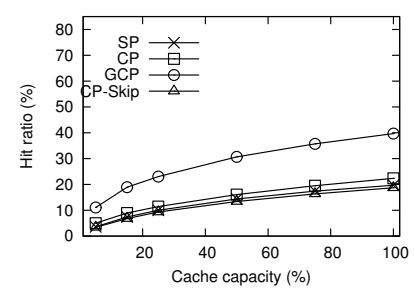

Dynamic

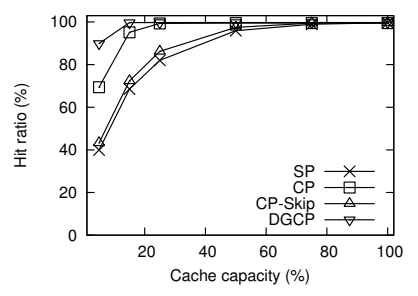

(a) Colorado

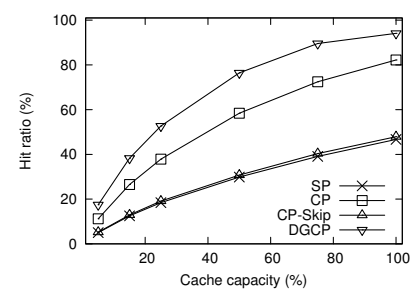

(b) New York
Figure 10: Hit ratio vs. cache capacity

cache capacty. In the static (dynamic) caching setting, GCP/DGCP can achieve a hit ratio of $84.28 \%(99.75 \%)$ and $39.70 \%(94.13 \%)$ for Colorado and New York, respectively. Observe that, in the dynamic caching setting, we can achieve a very high cache hit ratio with a small cache capacity. In the remaining experiments, we do not include the results for CP-Skip as CP-Skip and SP have similar hit ratios.

Figure 11 shows the impact of the cluster radius on the hit ratios of the methods. Observe that a smaller radius leads to a higher hit ratio. This is expected as a smaller radius implies that there are fewer unique query pairs in the workload.

In the static caching setting, GCP always outperforms its competitors, and CP is the second best, followed by CP-Skip and SP in the last tier. In the dynamic caching setting, DGCP clearly outperforms its competitors.

Figure 12 shows the impact of the number of clusters on the cache hit ratio. GCP/DGCP consistently outperforms the competitors, with a lead to the next-best competitor by at least $20 \%$. 


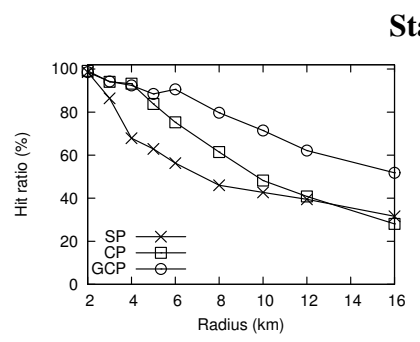

Static

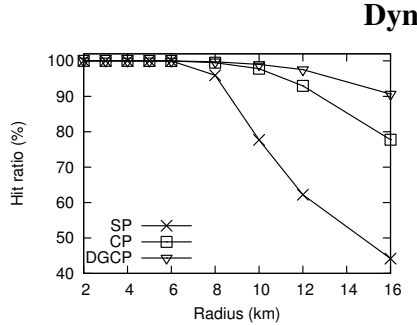

(a) Colorado

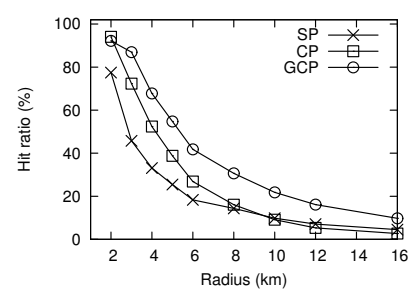

Dynamic

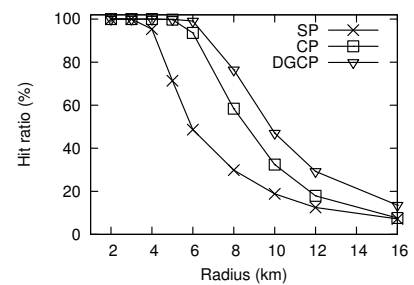

(b) New York
Figure 11: Hit ratio vs. cluster radius

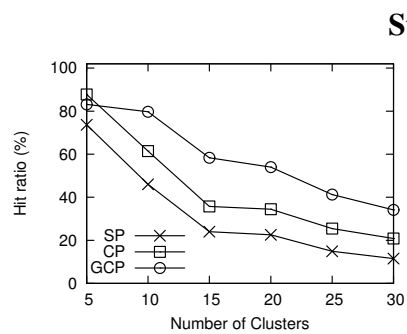

Static

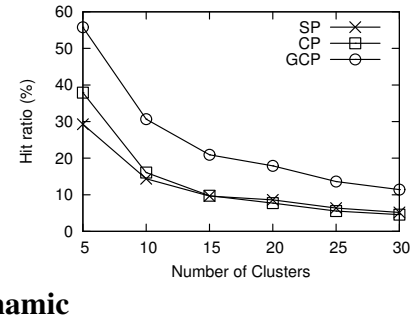

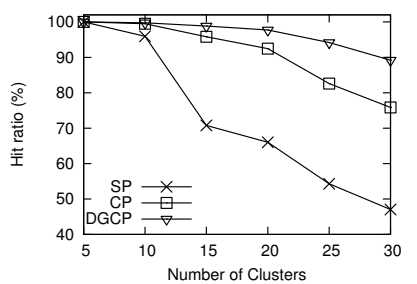

(a) Colorado

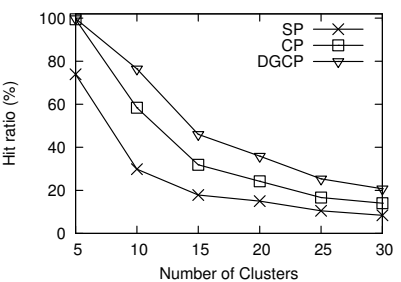

(b) New York
Figure 12: Hit ratio vs. number of clusters

\section{CONCLUSIONS}

In this paper, we exploit concise shortest paths to boost the cache hit ratio of shortest path queries in cache servers. First, we propose the notion of a generic concise shortest path that enables a tradeoff between the path size and the number of queries that can be answered by the path. Then we develop static and dynamic caching techniques for generic concise shortest paths.

Experimental results show that the hit ratios of our best methods (GCP and DGCP) are 10\%-40\% higher than that of competitors. Our methods are more robust with respect to the cache capacity.

As for the future work, we will study the maintenance of cache content with respect to dynamic traffic updates.

\section{Acknowledgements}

This work was supported by ICRG grant G-YN38 from the Hong Kong Polytechnic University and by a grant from the Obel Family Foundation.

\section{REFERENCES}

[1] Google Directions API. https://developers.google.com/ maps/documentation/directions/.

[2] MapQuest Open Directions API. http://developer.mapquest.com/web/products / open/directions-service.

[3] Web cache softwares. http://en.wikipedia.org/wiki/Web_cache.

[4] I. Abraham, A. Fiat, A. V. Goldberg, and R. F. F. Werneck. Highway dimension, shortest paths, and provably efficient algorithms. In SODA, pages 782-793, 2010.

[5] R. A. Baeza-Yates, A. Gionis, F. Junqueira, V. Murdock, V. Plachouras, and F. Silvestri. The impact of caching on search engines. In SIGIR, pages 183-190, 2007.

[6] G. V. Batz, R. Geisberger, D. Luxen, P. Sanders, and R. Zubkov. Efficient route compression for hybrid route planning. In MedAlg, pages 93-107, 2012.

[7] K. Button and D. Hensher. Handbook of Transport Systems and Traffic Control. Handbooks in Transportation Research Series. Elsevier Science, 2001.

[8] T. H. Cormen, C. E. Leiserson, R. L. Rivest, and C. Stein. Introduction to Algorithms. The MIT Press, 3rd edition, 2009.

[9] A. Dingle and T. Pártl. Web cache coherence. Computer Networks, 28(7-11):907-920, 1996.

[10] Q. Gan and T. Suel. Improved techniques for result caching in web search engines. In $W W W$, pages 431-440, 2009.

[11] R. Geisberger, P. Sanders, D. Schultes, and D. Delling. Contraction hierarchies: Faster and simpler hierarchical routing in road networks. In WEA, pages 319-333, 2008.

[12] H. Hu, J. Xu, W. S. Wong, B. Zheng, D. L. Lee, and W.-C. Lee. Proactive caching for spatial queries in mobile environments. In ICDE, pages 403-414, 2005.

[13] C. S. Jensen, H. Lahrmann, S. Pakalnis, and J. Runge. The INFATI data. arXiv preprint cs/0410001, 2004.

[14] K. Lee, W.-C. Lee, B. Zheng, and J. Xu. Caching complementary space for location-based services. In EDBT, pages 1020-1038. 2006.

[15] X. Long and T. Suel. Three-level caching for efficient query processing in large web search engines. In $W W W$, pages 257-266, 2005.

[16] E. P. Markatos. On caching search engine query results. Computer Communications, 24(2):137-143, 2001.

[17] E. J. O'Neil, P. E. O’Neil, and G. Weikum. The LRU-k page replacement algorithm for database disk buffering. In SIGMOD, pages 297-306, 1993.

[18] R. Ozcan, I. S. Altingövde, and Ö. Ulusoy. Static query result caching revisited. In $W W W$, pages 1169-1170, 2008.

[19] S. Podlipnig and L. Böszörményi. A survey of web cache replacement strategies. ACM Comput. Surv., 35(4):374-398, 2003.

[20] H. Samet, J. Sankaranarayanan, and H. Alborzi. Scalable network distance browsing in spatial databases. In SIGMOD, pages 43-54, 2008.

[21] Y. Tao, C. Sheng, and J. Pei. On k-skip shortest paths. In SIGMOD, pages 421-432, 2011.

[22] J. R. Thomsen, M. L. Yiu, and C. S. Jensen. Effective caching of shortest paths for location-based services. In SIGMOD, pages 313-324, 2012.

[23] P. Venetis, H. Gonzalez, C. S. Jensen, and A. Y. Halevy. Hyper-local, directions-based ranking of places. PVLDB, 4(5):290-301, 2011.

[24] B. Zheng and D. L. Lee. Semantic caching in location-dependent query processing. In SSTD, pages 97-116, 2001.

[25] Y. Zheng, L. Zhang, X. Xie, and W.-Y. Ma. Mining interesting locations and travel sequences from GPS trajectories. In $W W W$, pages 791-800, 2009.

[26] A. D. Zhu, H. Ma, X. Xiao, S. Luo, Y. Tang, and S. Zhou. Shortest path and distance queries on road networks: towards bridging theory and practice. In SIGMOD, pages 857-868, 2013. 